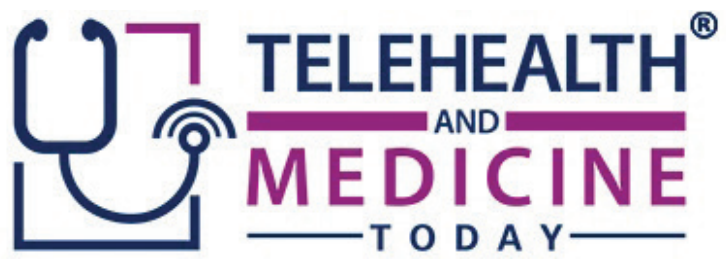

\title{
VA Video Telehealth and Training Programs During the COVID-19 Response
}

\author{
Rhonda Johnston, Ph.D., BC-FNP, BC-ANP ${ }^{1}$, Rita F. Kobb, MSN, APRN-BC ${ }^{2}$, \\ Claire Marty, MN, RN ${ }^{3}$, Padraic McVeigh, RN, MSN ${ }^{4}$
}

Affiliation: ${ }^{1}$ Director, VA Office of Connected Care Quality and Training Division, U.S. Department of Veterans Affairs, Office of Connected Care, Quality and Training Division; ${ }^{2}$ Telehealth Training Team Lead, VA Office of Connected Care Quality and Training Division, U.S. Department of Veterans Affairs, Office of Connected Care, Quality and Training Division; ${ }^{3}$ National Quality Lead, VA Office of Connected Care Quality and Training Division, U.S. Department of Veterans Affairs, Office of Connected Care, Quality and Training Division; ${ }^{4}$ Quality Manager, VA Office of Connected Care Quality and Training Division, U.S. Department of Veterans Affairs, Office of Connected Care, Quality and Training Division

Corresponding Author: Johnston L. Rhonda, Email: rhonda.johnston@va.gov

Keywords: COVID-19 PANDEMIC, Surgery, Telehealth, VA, Vetean's Administration, Video

Section: Training Tools for Health Professionals \& Patients

Objective: The U.S. Department of Veterans Affairs has a robust telehealth program supported by a training infrastructure for VA clinicians and staff. In fiscal 2019, over 909,000 $V A$ patients received a portion of their VA care through telehealth, and over $60 \%$ of VA's primary and mental health care providers participated in one or more Clinical Video Telehealth (CVT) appointments to a patient's home.

In March 2020, during the initial spread of COVID-19, Veterans Health Administration leadership issued guidance for providers to transition from in-person care to virtual modalities for routine care not requiring a physical encounter. VA's comprehensive training infrastructure helped providers quickly become capable of delivering CVT care to meet the needs of their patients, and the VA Office of Connected Care Quality and Training (QT) Division adjusted the program as providers' needs evolved.

Design: As clinical staff faced new and increasing demands to respond to needs during the pandemic, the QT Division streamlined existing training programs to deliver essential content and enable providers to acquire necessary telehealth skills more quickly. This included reducing the number of core courses required to become telehealth-capable from four to two by introducing an integrated 
course. The QT Division reorganized and edited content from other accredited courses to convey key topics. To shorten courses for providers with full schedules, the QT Division removed accreditation from some courses, such as the COVID-19 VA Video Connect for Providers course.

Results: The QT Division's pandemic response resulted in over 80,000 new course completions in the first month and over 20\% more primary care and mental health providers conducting CVT visits in the first five months. During this period, the number of CVT appointments increased by over 1,000\%.

Conclusion: VA's telehealth training infrastructure, implemented by the QT Division, enabled VA to enter the COVID-19 public health emergency fully prepared for the rapid growth in telehealth care. As CVT became increasingly important during the COVID-19 response, the QT Division made necessary curriculum adjustments to meet provider and patient needs.

\section{INTRODUCTION}

Video telehealth has played a crucial role in the U.S. Department of Veterans Affairs' response to the COVID-19 pandemic. As VA providers transitioned to providing remote care for their patients, VA experienced exponential growth in telemedicine - particularly in the use of synchronous video telehealth care, known at VA as Clinical Video Telehealth (CVT). Monitoring of telehealth services showed that CVT visits to patients' homes and other non-VA sites expanded significantly over the first five months of the COVID-19 pandemic, with an increase in weekly visits of over $1,000 \%$ since early March.

The increased use of CVT has been supported by the telehealth training infrastructure that VA has developed for clinical staff. The telehealth training program was in place before the COVID-19 pandemic and expanded and adjusted to meet providers' needs during the pandemic. This article outlines VA's telehealth training infrastructure and demonstrates how it enabled CVT's growth and critical role in response to the COVID-19 pandemic while maintaining positive evaluations from both VA staff and patients.

\section{FOUNDATIONS OF VA TELEHEALTH TRAINING AND DELIVERY}

\section{Training Infrastructure}

Before the COVID-19 pandemic, expanding telehealth care for Veterans was a key goal of the Veterans Health Administration. A core element of this goal was implementing evidence-based training programs to enable VA clinical staff to deliver telehealth care. The Office of Connected Care, which oversees the VHA telehealth program, began implementing training programs in 2004. The Connected Care Quality and Training (QT) Division developed the training programs based on literature that indicated that education and training programs for health care staff improved clinical outcomes for patients. ${ }^{1,2,3}$

VA's training model includes role-based competency programs, preceptor-supported training at the point of performance, and technology accessibility training. The main platform for these courses is the Connected Care Academy (Academy), which operates on the Blackboard learning management system (LMS). Through the Academy, the QT Division offers self-paced courses as well as live events to increase accessibility and distribution of training content. All VHA staff have access to the Academy, and non-VA staff can access it through a self-registration process. The QT Division records course completions and issues certificates for continuing education units (CEUs) to users, as desired. 
Currently, the Connected Care Academy has 118 self-paced courses. This includes 15 to 20 courses for each of VA's core telehealth modalities: CVT, Remote Patient Monitoring Home Telehealth, and Asynchronous Store-and-Forward Telehealth. The Academy also includes approximately 20 technology courses, 35 telespecialty courses, and five courses on telehealth care team roles. The self-paced courses are complemented by numerous live training sessions, forums, and office hours; personalized skill assessments; practicum projects; and competency checklists.

All staff must take four core courses to qualify to deliver telehealth care. Also, each telespecialty area and care team role has its own set of required and elective courses. Staff with licensure are expected to take self-paced courses on a regular basis to maintain status. For any accredited course, staff are required to score $80 \%$ or higher on a knowledge-based test to earn CEUs. Staff are alerted to new courses by assignment in Blackboard and through various staff outreach channels.

QT Division staff evaluate the training programs using feedback from training participants, VHA faculty, and subject matter experts. Course feedback - which includes participants' responses on how they liked the learning process, the extent to which they had gained knowledge and skills, and their ability to perform new skills on the job - is reviewed weekly. Evaluation also involves consideration of training participants' demographic data (which includes facility location, job role, and specialty) and post-course surveys for content comprehension. The QT Division reviews all courses at least once a year, and analysis of the results of this review informs the next round of content revisions for those courses. These course revisions frequently involve adjusting content to reflect practice changes or technology updates and streamlining course offerings. Most courses undergo significant content updates every other year.

\section{VA Telehealth Before the Pandemic}

The training program enabled a growing number of VHA clinical staff to deliver telehealth care, which supported VA's goal of expanding telehealth to reach more patients. In fiscal year 2019, VA clinical staff, which included providers, nurses, social workers, and pharmacists, completed an average of approximately 6,400 telehealth training courses per month through the Academy. ${ }^{4}$ As a result, a large proportion of VA primary care and mental health providers were already capable of delivering telehealth care at the onset of the COVID-19 outbreak. By the end of fiscal 2019 , over $60 \%$ of VA's roughly 25,000 primary care and mental health care providers had conducted at least one CVT visit to patients' homes or other off-site locations. ${ }^{4}$

The telehealth training infrastructure helped VA develop a well-established CVT program prior to the pandemic. In fiscal 2019, over 909,000 patients received a portion of their VA care through telehealth, representing a $16 \%$ increase from the previous fiscal year. Of those patients, over 99,000 had at least one CVT visit to the home or another off-site location, a 246\% increase from fiscal 2018. In total, VA conducted over 294,000 CVT visits to patients' homes or off-site locations in fiscal 2019, primarily through VA Video Connect, VA's main CVT platform. ${ }^{5}$

Before the COVID-19 pandemic, however, some VA clinical staff had still exhibited skepticism about telehealth. In the open-ended comments of the post-training surveys, VA staff occasionally voiced concerns about the appropriateness of telehealth for particular types of care, the lack of technology amid the patient population or in the 
clinic itself (e.g., in rural areas), or conflicting priorities that diverted staff time from completing training courses. The QT Division received anecdotal feedback from some VA providers expressing concerns that new technologies might malfunction or cause them to appear less authoritative, experienced, or trustworthy to their patients.

\section{COVID-19 RESPONSE - TRAINING EXPANSION AND EVOLUTION}

In March 2020, during the initial spread of COVID-19, VHA leadership issued guidance to providers to transition from in-person care to virtual modalities for routine care not requiring a physical encounter. The comprehensive training infrastructure helped providers quickly become capable of delivering CVT care to meet the needs of their patients, and the QT Division adjusted the program as providers' needs evolved.

Core elements of the training infrastructure proved vital during the emergency response. The Academy LMS enabled providers to access the CVT core curriculum and other courses on their own schedules. Before the pandemic, the QT Division had expanded the Academy to enable clinical staff to access courses from any device and from any location, which helped teleworking providers quickly access courses from home.

The QT Division used the existing course evaluation process to assess and meet provider needs during the COVID-19 pandemic. As clinical staff faced new and increasing demands to respond to the pandemic, QT Division staff streamlined existing training programs to deliver essential content. This included introducing an integrated course to reduce the number of core courses staff were required to take to become telehealth-capable from four to two. The QT Division reorganized and edited content from other accredited courses to convey key topics.
To shorten courses for providers with full schedules, the QT Division created shorter, unaccredited versions of some courses, such as the COVID-19 VA Video Connect for Providers course. This change enabled providers to acquire necessary telehealth skills more quickly. The QT Division also added new courses and made relevant courses mandatory, as necessary, to meet evolving needs, including courses focused on CVT care and COVID-19. Training staff have prioritized assessment of all training courses and analyzed over 8,000 learner comments within the first two months of the pandemic to inform future revisions.

To complement the self-paced courses, QT Division staff also held instructor-led live training sessions over virtual platforms, including Skype and Adobe Connect. These live forums gave providers, especially those new to telehealth, the opportunity to interact with training staff in real time on topics related to COVID-19 and telehealth care. Trainers tailored these forums to meet the content and scheduling needs of a national clinical workforce spanning over 150 VA facilities in five different time zones. From March 29 to May 17, 2020, the QT Division held 156 forums, which were attended by over 3,700 clinical staff. ${ }^{6}$

\section{OUTCOMES AND EVALUATIONS Growth in Provider CVT Capability}

The number of training course completions increased dramatically during the COVID-19 response. In March 2020, VA staff completed nearly 87,000 total training courses - more course completions than in the previous 12 months combined (about 83,000). The primary VA Video Connect training had over 24,000 course completions in March. Since March, completions for all courses have remained much higher than average: over 42,000 in April, 18,000 in May, and 15,000 in June. ${ }^{7}$ 


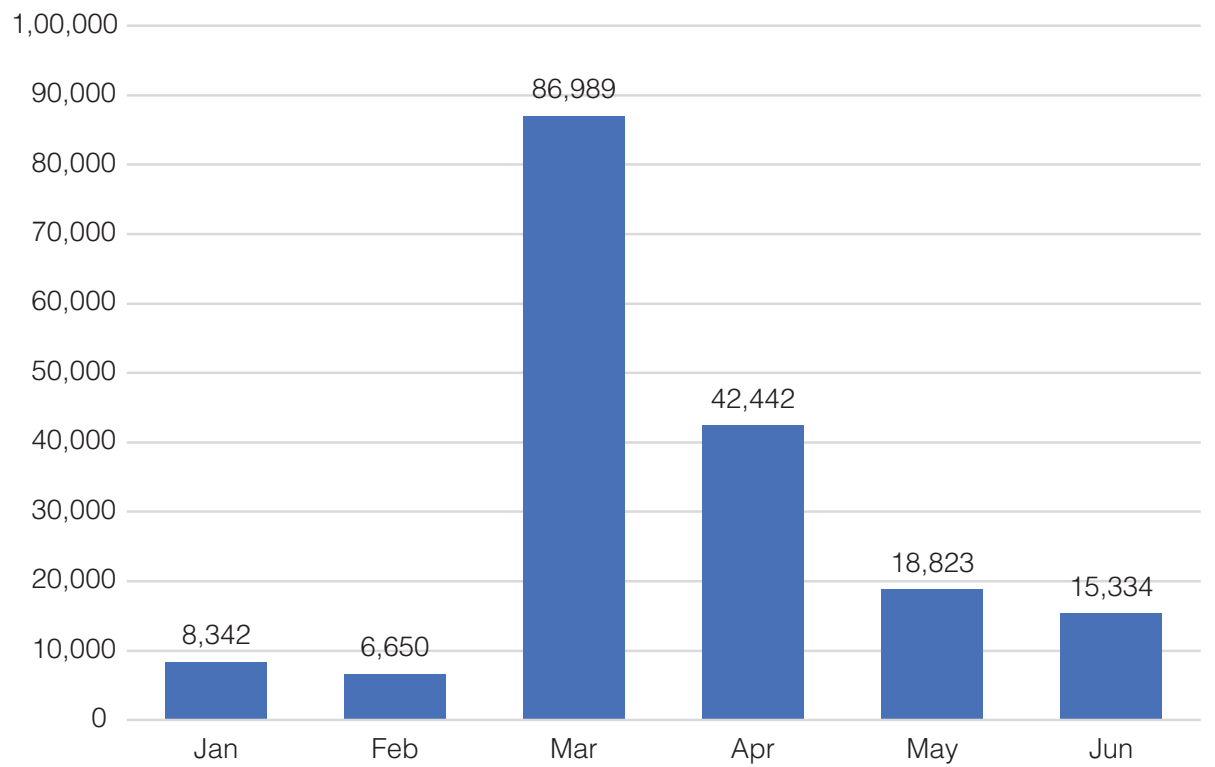

Figure 1-Total telehealth training course completion in 2020.

The rise in course completions corresponded with an increase in the number of VA primary care and mental health care providers conducting CVT visits. At the end of February 2020, 66.3\% of VA primary care providers and $67.6 \%$ of VA mental health care providers used CVT to conduct visits to patients' homes or other off-site locations. From February 29 to August 2, 2020, the number of primary care providers who used CVT grew by $21.8 \%$, and the number of mental health care providers who used CVT grew by $24.4 \%$. Currently, about nine in 10 of VA's primary care and mental health care providers have conducted at least one CVT visit to a patient's home or another off-site location. ${ }^{8}$

\section{Increase in CVT Visits to Home and Off-Site}

In the first week of March 2020, before widespread stay-at-home orders in response to COVID-19, VA conducted 10,687 CVT visits to the home or another off-site location. This number quickly increased: VA reported weekly averages of 91,686 such visits in April, 108,414 visits in May, 138,275 visits in June, and 146,748 visits in July. ${ }^{9}$
The reach of the CVT program in fiscal 2020 has far exceeded that of fiscal 2019, which was a record-setting year for VA telehealth. In June 2020, VA providers conducted 592,608 total CVT visits to patients' homes or other off-site locations, more than double the number conducted in all of fiscal 2019. ${ }^{9,10}$ Additionally, during the first 10 months of fiscal 2020 (from October 2019 through July 2020), over 700,000 VA patients received at least one CVT visit to their homes or other off-site locations. ${ }^{10}$ This was over seven times as many patients as seen in all of fiscal 2019.

\section{Clinical Staff and Patient Evaluations}

Clinical staff evaluations of the training program remained overwhelmingly positive during the COVID-19 response. Through the third quarter of fiscal 2020, average scores for course knowledge (97.3\%), relevance (93.8\%), application (93.9\%), scope (93.8\%), and satisfaction (93.2\%) were all higher than the same scores for fiscal 2019. Learner satisfaction scores were above $90 \%$ for all newly developed content and core telehealth courses. In evaluations of the live forums, an overwhelming majority of 


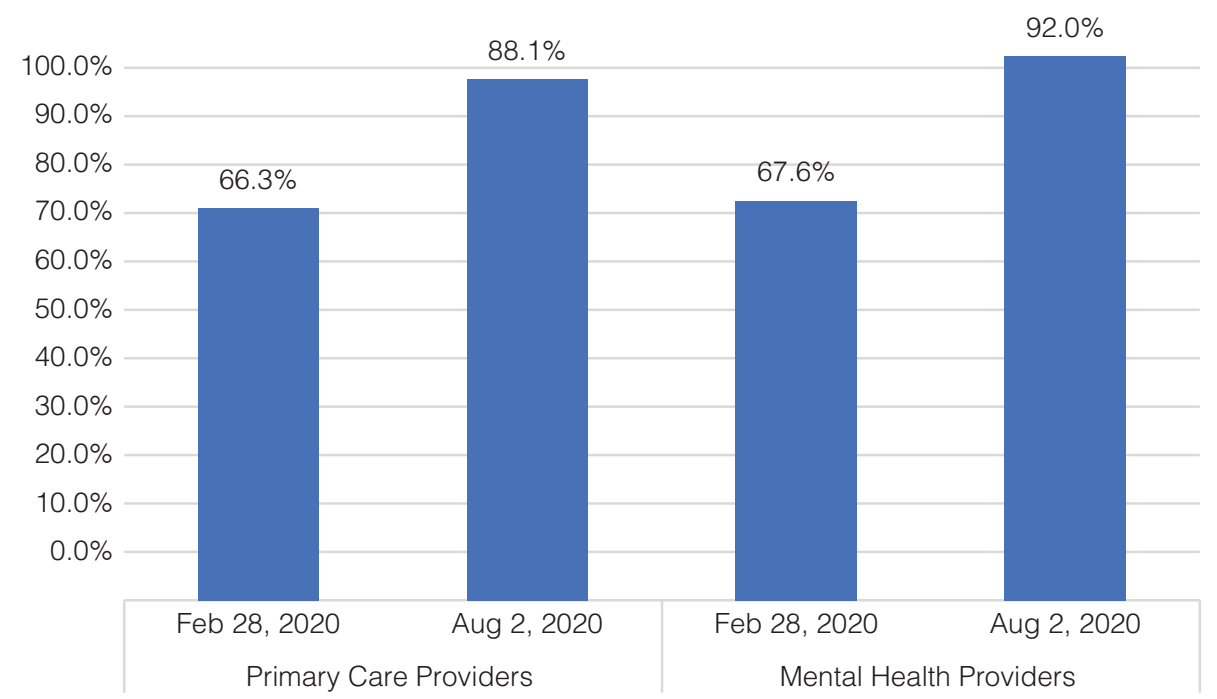

Figure 2-Percentage of VA providers who conducted one or more CVT visits to a patient's home or another off-site location.

attendees said that sessions provided them with useful information for their telehealth roles (94.3\%), proved helpful to them (93.5\%), and taught them new knowledge and skills $(92.7 \%){ }^{8}$

In addition, VA telehealth training sessions and forums have promoted positive telehealth experiences for patients who received care through CVT. The results of a survey conducted from October 2019 through May 2020 showed that VA patients overwhelmingly were satisfied with their CVT care. The survey asked respondents to rate aspects of their CVT care on a scale of one to five, with five being the best possible score. In the survey, $86.1 \%$ of patients scored their overall satisfaction with their CVT visit as a four or a five. Over $93 \%$ of respondents rated their providers with a four or a five for listening in a caring manner, explaining things in an easy-tounderstand manner, and putting them at ease by clearly explaining each step of the CVT appointment. Additionally, over $86 \%$ of patients gave the same scores for being able to easily access VA Video Connect and being able to clearly see and hear the provider during the visit. ${ }^{9}$ These high scores indicated that CVT care maintained high satisfaction among patients as a treatment option.

\section{Challenges to Continued Telehealth Growth}

Although the QT Division continues to evaluate the training program on a weekly basis, there remain systemic challenges to the training program, and to telehealth more broadly. VA administers its training program to a national audience, but specific locations may have different capabilities and needs. In smaller VA clinics, and in many of the communities VA serves, a lack of relevant technology continues to hamper telehealth adoption and makes some of the courses and programs offered by the training team irrelevant to local staff. In other instances, the national training program does not address very specific patient or local situations and relies on local expertise to fill in the gaps. As telehealth care grows at VA, its continued success relies on busy practitioners to set standards, provide guidance, and support the implementation of new training programs and telehealth offerings at each VA location. The QT Division is taking steps to meet localized needs by expanding outreach programs to provide VA 


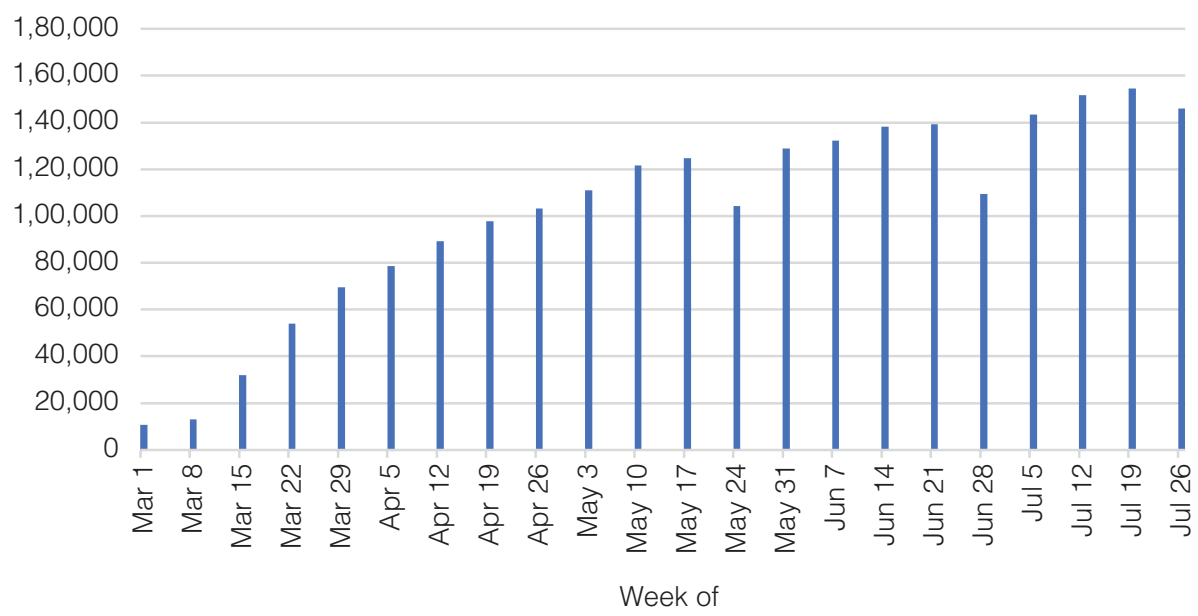

Figure 3-Total CVT visits to home or off-site location by week, March-July 2020.

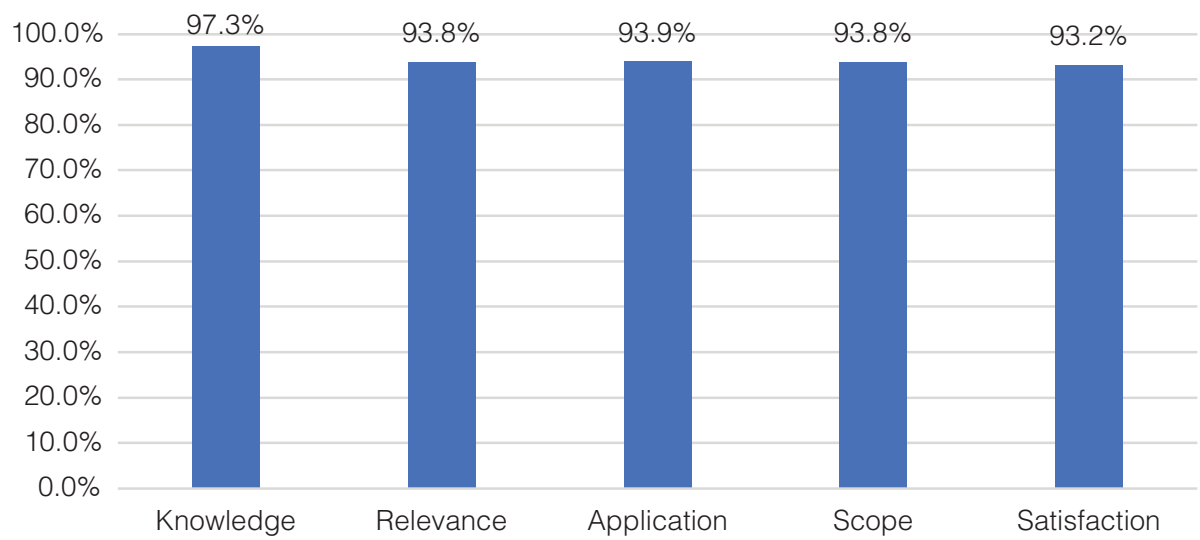

Figure 4-Telehealth course provider evaluation metrics, October 2019 - June 2020.

clinical staff with the full complement of telehealth tools and resources. Additionally, office hours and live forums administered by QT Division trainers can address more specific questions and needs in real time.

VA continues to encounter barriers to telehealth adoption by VA patients - primarily, the digital divide that exists for many VA patients who lack internet access or the technology fluency needed for telehealth. This challenge requires broad institutional investment in broadband expansion. VA is engaging in public-private partnerships that bridge the digital divide and expand telehealth access to underserved communities. While some skepticism about telehealth persists among patients and VA clinicians, its central role during VA's COVID-19 response has demonstrated its potential benefits to many. The pandemic response has illustrated the need for and the value of continued investment in telehealth care.

\section{CONCLUSION}

VA's telehealth training infrastructure, implemented by the Office of Connected Care's QT Division, enabled VA to enter the COVID19 public health emergency fully prepared for the rapid growth in telehealth care. As CVT 


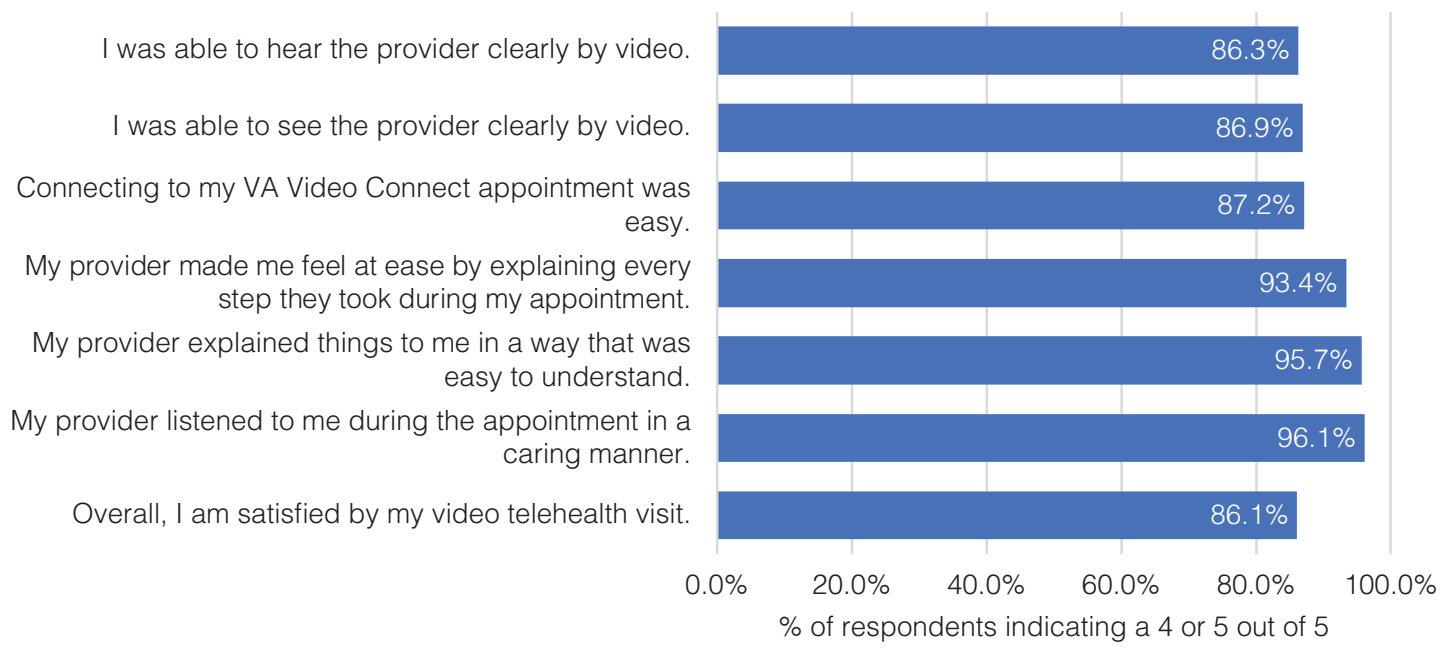

Figure 5-VA patient telehealth survey results, October 2019 - May 2020.

became increasingly important during the COVID-19 response, the QT Division made necessary curriculum adjustments to meet provider and patient needs.

The QT Division continues to monitor and adjust training content and delivery based on evaluations by clinical staff and patients. Quality oversight remains a focal point for VA and will enable telehealth care to grow while meeting patient needs safely and effectively. Future program evaluations will remain critical to the continued growth and success of telehealth.

The QT Division will continue to prepare all clinical staff to provide care virtually, through CVT and other modalities. VA's goal is to ensure every ambulatory care VA provider is capable of delivering video telehealth care, including providers in key telespecialty areas such as infectious disease, mental health, and pulmonary care. VA can meet this goal with continued emphasis on training and full integration of telehealth into the VA health system.

\section{References}

1. Weaver SJ, Dy SM, Rosen MA. Teamtraining in healthcare: a narrative synthesis of the literature. BMJ Qual Saf [Internet]. 2014;23:359-372. Available from: https:// qualitysafety.bmj.com/content/23/5/359 DOI: 10.1136/bmjqs-2013-001848

2. Garzonis K, Mann E, Wyrzykowska A, Kanellakis P. Improving patient outcomes: Effectively training healthcare staff in psychological practice skills: a mixed systematic literature review. Europe's Journal of Psychology [Internet]. 2015;11(3):535-556. Available from: https://ejop.psychopen.eu/index.php/ ejop/article/view/923 DOI: 10.5964/ejop. v11i3.923

3. Karami A, Farokhzadian J, Foroughameri G. Nurses' professional competency and organizational commitment: Is it important for human resource management? PLoS ONE. 2017;12(11): Article e0187863. Available from: https://journals.plos.org/plosone/ article? $\mathrm{id}=10.1371 /$ journal.pone. 0187863 DOI: 10.1371/journal.pone.0187863

4. McVeigh P. Primary Care and Mental Health Providers Using CVT to Home. U.S. Department of Veterans Affairs, Veterans Health Administration, VSSC Reports; 2020.

5. Peters J. VHA Talking Points - Telehealth, Fiscal 2019. U.S. Department of Veterans Affairs, Veterans Health Administration; 2019.

6. McVeigh, P. Veteran Satisfaction, Telehealth at Home or Mobile Appointment Survey. 
VSignals, Veterans Experience Office. U.S. Department of Veterans Affairs; 2020.

7. McVeigh P. Telehealth Virtual Forums Dashboard. U.S. Department of Veterans Affairs Office of Connected Care, Quality and Training Division; 2020 [cited 2020 June 24].

8. McVeigh P. Quality and Training Dashboard. U.S. Department of Veterans Affairs Office of Connected Care, Quality and Training Division; 2020 [cited 2020 July 22].

9. Stressel P. Video Visits to Home or Off-Site. U.S. Department of Veterans Affairs, Office of Connected Care; 2020.

10. Galpin K. VA Telehealth Talking Points for White House Domestic Policy Council. U.S.
Department of Veterans Affairs, Office of Connected Care; 2020.

Copyright Ownership: This is an open access article distributed in accordance with the Creative Commons Attribution Non Commercial (CC BY-NC 4.0) license, which permits others to distribute, adapt, enhance this work non-commercially, and license their derivative works on different terms, provided the original work is properly cited and the use is non-commercial. See: http://creativecommons.org/licenses/ by-nc/4.0 